\title{
Molecular fluctuations as a ruler of force-induced protein conformations
}

Andrew Stannard ${ }^{1 *}$, Marc Mora ${ }^{1 *}$, Amy E.M. Beedle ${ }^{1 *}$, Marta Castro-López ${ }^{1}$, Stephanie Board ${ }^{1}$, Sergi Garcia-Manyes ${ }^{1,2 \uparrow}$

${ }^{1}$ Department of Physics, Randall Centre for Cell and Molecular Biophysics and London Centre for Nanotechnology, King's College London, Strand, WC2R 2LS London, United Kingdom.

${ }^{2}$ The Francis Crick Institute, 1 Midland Road, London NW1 1AT, London, UK.

*These authors contributed equally to the work

"Corresponding author: sergi.garcia-manyes@kcl.ac.uk 


\begin{abstract}
Molecular fluctuations directly reflect the underlying energy landscape. Variance analysis can probe protein dynamics in several biochemistry-driven approaches, yet measurement of probeindependent fluctuations in proteins exposed to mechanical forces remains only accessible through steered molecular dynamics simulations. Using single molecule magnetic tweezers, here we conduct variance analysis to show that individual unfolding and refolding transitions occurring in dynamic equilibrium in a single protein under force are hallmarked by a change in the protein's end-to-end fluctuations, revealing a change in protein stiffness. By unfolding and refolding three structurally distinct proteins under a wide range of constant forces, we demonstrate that the associated change in protein compliance to reach force-induced thermodynamically-stable states scales with the protein's contour length, in agreement with the sequence-independent FJC model of polymer physics. Our findings will help probe the conformational dynamics of proteins exposed to mechanical force at high resolution, of central importance in mechanosensing and mechanotransduction.
\end{abstract}




\section{Introduction}

Mechanical unfolding of proteins regulates a variety of biological processes, encompassing muscle elasticity $^{1}$, focal adhesion mechanosensing ${ }^{2,3}$ and nuclear translocation ${ }^{4}$. Whether working as monomers or in form of polyproteins ${ }^{5}$, mechanical unfolding provides an efficient shock absorbing strategy that works as a safety mechanism to preserve the mechanical integrity of adhesive and mechanotransducing protein complexes ${ }^{6}$. Several strategies -including the protein's intrinsic topology, the introduction of point mutations, ligand binding, the pulling direction or the dynamic rupture/reformation of covalent bonds - have been reported to govern the mechanical resistance of proteins in their folded form ${ }^{7}$. Once unfolded, mechanically stretched proteins exhibit previously hidden sites to the solvent, which become suddenly reactive to protein binding or post-translational modifications, ultimately affecting protein elasticity ${ }^{8}$. Given the emerging conformation-dependent functionality ${ }^{9}$, it is enticing to experimentally capture the mechanical properties of the different protein forms, collectively defining the rich conformational repertoire of proteins under force.

Single molecule nanomechanical experiments have provided a detailed in-vitro insight of the molecular determinants governing the conformational dynamics of proteins under force ${ }^{8,10}$. Collectively, these measurements have concentrated on providing an extensive characterization of the mechanical stability of the native state of proteins, and on capturing the kinetics and out-ofequilibrium transition paths underpinning individual unfolding and refolding trajectories ${ }^{11}$. By contrast, a thorough experimental description of the extended states — often perceived as a single, featureless, compliant conformation — has comparatively received much less attention. This largely stems from the lack of experimental approaches able to capture the evolution of protein fluctuations with force, which emerge naturally from a general and rather simplified picture of the folding energy landscape that defines the behaviour of an entropic ideal polymer under force (predicted by models of protein elasticity ${ }^{12}$ e.g. the freely-jointed chain (FJC) or worm-like chain (WLC)) added to an enthalpic, short-range attractive contribution (e.g. Morse or Lennard-Jones potential) ${ }^{13}$. In the absence of force, proteins populate this latter energy basin, which defines the native state of the protein (Fig. 1). The application of a constant force tilts the 1D energy landscape and creates a new energy minimum that corresponds to the protein's unfolded state. Increasing the stretching force lowers the energy of the unfolded well and displaces its position toward increasingly extended conformations, as predicted by the FJC/WLC model. As a natural consequence, the width of the unfolded minimum decreases with increasing the stretching force. Hence, the measurement of the length fluctuations as a function of force should provide an accurate and direct measurement of protein stiffness. Despite its theoretical simplicity, a systematic experimental quantification of the dependency of protein stiffness with protein extension has evaded characterization, mainly due to the technical inability to capture the natural fluctuation modes of the protein when exposed to the wide range of stretching forces that fingerprint its entire conformational space, encompassing both the native and unfolded conformations.

Here we employ single molecule magnetic tweezers ${ }^{14-17}$, which enable (i) capturing of the unfolding/refolding trajectories of the same single protein over extended (hour-long) periods of time with high stability ${ }^{15}$, (ii) under a wide range of constant forces (2-100pN), (iii) in passive clamp mode (hence avoiding potential masking of the protein fluctuations due to the active electronic feedback ${ }^{18}$ ), (iv) in the absence of long tethers (which necessarily add additional inherent fluctuations to the protein construct $\left.{ }^{19}\right)$, and, most importantly, $(v)$ in an experimental set-up where the measured stiffness is dominated by the intrinsic stiffness of the protein-of-interest (and not the pulling device, such as e.g. the AFM cantilever ${ }^{20-22}$, Fig. S1 and SI text) to capture the change in compliance upon mechanical unfolding/refolding of individual proteins, in both monomeric and polymeric forms. We first apply variance analysis to measure the change in noise (end-to-end fluctuation) as the protein 
undergoes multiple unfolding and refolding transitions under force in equilibrium. Furthermore, we measure the change in protein compliance as the protein is unfolded and held stretched under a wide range of forces to directly compare our experimental results with an analytical solution relating the change in protein's compliance as a function of its extension change derived from the FJC model of polymer elasticity. We test the consistency our approach by studying the unfolding and refolding dynamics of three structurally distinct proteins such as the model protein $L$ and the talin and nesprin mechanosensors. Combined, our experiments enable direct observation of the change in protein stiffness upon mechanical unfolding and over a continuum range of force-induced conformations in thermodynamic equilibrium, and demonstrate that molecular fluctuations are a reliable reporter of force-induced protein conformations.

\section{Results}

\section{A change in compliance fingerprints single (un)folding events}

Using single molecule magnetic tweezers, we monitored the unfolding and refolding trajectories of an individual protein $\mathrm{L}(\mathrm{PL})$ monomer bracketed between two mechanically rigid Ig32 titin domains either side. The resulting (Ig32) 2 -PL-(Ig32) 2 polyprotein is covalently attached to the glass substrate through a Halo-Tag on the $\mathrm{N}$-terminus, and to the paramagnetic bead via biotin-streptavidin binding on the C-terminus (Fig. 2A and SI text). Using a force quench protocol, we first applied a constant force of $38 \mathrm{pN}$, which elicited the unfolding of the individual PL monomer, fingerprinted by a step elongation of the protein by $\Delta\langle x\rangle=16.8 \mathrm{~nm}^{15}$. We note that, at this force, we hardly ever captured the unfolding of the much stiffer Ig32 domains. After 60 seconds, the force was quenched down to $8.1 \mathrm{pN}$-the force, $F_{0.5}$, at which PL exhibits a $50 \%$ folded probability- and held constant for 60 minutes. During this period of time, we observed the stochastic unfolding and refolding of the PL monomer (hallmarked by an average step-change in extension of $\Delta\langle x\rangle=10.3 \pm 0.3 \mathrm{~nm}$ ) occurring with approximate equal probability ${ }^{15}$, Fig. 2B. The protein was finally stretched back again at $38 \mathrm{pN}$ to ensure that, over this long pulling time, the protein had not misfolded.

To experimentally measure changes in the mechanical properties of $\mathrm{PL}$ each time the monomer hops between the folded and unfolded states, we first consider that the measured stiffness, $k_{\mathrm{M}}$, of the entire series construct (combining the stiffness of $\mathrm{PL}, k_{\mathrm{P}}$, and the rest of the construct, $k_{\mathrm{C}}$ ) is given by $1 / k_{\mathrm{M}}=1 / k_{\mathrm{P}}+1 / k_{\mathrm{c}}$. As such, upon un/folding, the change in measured stiffness does not correspond to the change in PL stiffness $\left(\Delta k_{\mathrm{M}} \neq \Delta k_{\mathrm{P}}\right)$. If instead we consider compliance -the inverse of stiffness, $c=1 / k$ - we have the simple situation where $c_{M}=c_{P}+c_{C}$ and, crucially, the change in measured compliance is the change in PL compliance $\left(\Delta c_{M}=\Delta c_{P}\right)$. In the simple case of the classic Hookean spring, equipartition theory implies that constant (forceindependent) compliance is given by

$$
c=\beta \sigma^{2}
$$

where $\beta=1 / k_{\mathrm{B}} T$ is the inverse of thermal energy, and $\sigma^{2}=\left\langle x^{2}\right\rangle-\langle x\rangle^{2}$ is the variance in end-to-end length, $x$. The trivial consequence is that the length fluctuations are force independent. By contrast, in the case of an entropic spring, where the average length $\langle x\rangle$ is non-linear with force, compliance is force-dependent since $c \equiv \mathrm{d}\langle x>/ \mathrm{d} F$. Even in this case, Eq. 1 still holds true (SI text), implying that fluctuations are force-dependent, being lower at higher forces, and can be used to determine compliance of non-linear springs such as proteins. Combining these two arguments, we see that the change in PL compliance can be found from the change in measured variance, $\Delta c_{\mathrm{P}}=\beta \Delta \sigma_{\mathrm{M}}{ }^{2}$.

We next processed the hopping trajectory of Fig. 2B by flattening individual sections to remove the effects of slow timescale drift of the magnetic bead relative to the reference bead $(<10$ $\mathrm{nm}$ /hour average). Applying Eq. 1 to the resulting trajectory (Fig. $2 \mathrm{C}$ ) enables direct measurement of 
the protein's compliance (Fig. 2D) corresponding to the unfolded and folded states before and after each individual (un)folding transition ${ }^{23}$ (SI text). By averaging the compliance for both, the nativelyfolded (blue) and unfolded (red) states underpinning the 13 individual transitions in Fig. 2, we obtained an increase in protein compliance $\Delta c=0.86 \pm 0.09 \mathrm{~nm} / \mathrm{pN}$ upon PL unfolding (see also Fig. S3). Conceptually, this increase in compliance corresponds to the transition of the protein, at a constant force $(8.1 \mathrm{pN})$, from the narrow basin defining the native state to the much-broader well defining the unfolded state of the protein (Fig. 1).

\section{Protein stiffness evolution in a dynamically folding polyprotein}

Having obtained a measurable change in protein compliance upon mechanical unfolding of an individual protein monomer, we conjectured that, in the more complex case of a polyprotein (Fig. $3 \mathrm{~A}$ ), we might be able to single out the (un)folding of each individual domain by directly measuring the fluctuation variance of the entire construct at a given time and under constant force. To test this hypothesis, we repeated our force-quench protocol on a $\mathrm{PL}_{8}$ polyprotein (Fig. 3B). The long quench pulse at $8.1 \mathrm{pN}$ exhibited complex dynamics, underpinning the stochastic unfolding/refolding transitions of each distinct protein monomer forming the polyprotein chain (Fig. 3C). Measuring the variance of the overall fluctuations of the polyprotein construct at each state (number of unfolded domains) level revealed a linear correlation between the construct's compliance and the number of unfolded domains (Fig. 3D and Fig S4). Notably, the slope of the trend ( $\Delta c$ /domain $=0.81 \pm 0.15$ $\mathrm{nm} / \mathrm{pN}$ ) is in very good agreement with that obtained for the monomeric protein (Fig. 2D). These results further highlight that the different monomers forming a polyprotein chain fold independently, implying that the folding dynamics of an individual monomer is independent of the composition of the rest of the multidomain construct ${ }^{24,25}$. Most importantly, our results reveal that the dynamic change in compliance of a multi-protein construct can be used as a direct proxy to identify the number of folded (and unfolded) modules within an actively folding homopolyprotein at a given time.

\section{The FJC model captures the compliance changes with extension}

In the equilibrium experiments reported thus far, the pulling force was held constant to acquire equally sufficient statistics of the two protein states as the protein hopped between the unfolded/folded conformations. To systematically study the effect of force on protein compliance, we expanded the range of probed forces by conducting successive unfolding/refolding cycles on the same single protein monomer (Fig. 2A), where the unfolding/refolding force was varied in the range 4.3-22 pN (Figs. 4A\&B). We then measured the relative change in the fluctuation variance (and the related change in protein compliance) for each particular force (Fig. 4C\&D). These results clearly demonstrate that, when PL is mechanically unfolded, the greater the change in average extension (due to higher force being applied), the lesser the change in compliance. Finally, our resulting data on the extension-dependent change in compliance was compared to the prediction by the FJC model - which has an analytical solution for $\Delta<x>$ and $\Delta c$ (SI text). Our experimental data, including both the monomer and polymer measurements, quantitatively agree with the FJC model (Fig. 4D), confirming that the change in compliance (ultimately measured through the protein's end-to-end length fluctuations) is reduced as the pulling forces are increased, and that the findings should be general and independent of the studied protein since the FJC has no consideration for protein structure.

\section{The rapid hopping of talin is mirrored by molecular fluctuations}

A closer look to the analytical expression obtained from the FJC model (SI text) reveal that both the force-dependent extension change, and the force-dependent compliance change, are 
predicted to be linear with contour length increment. To experimentally test this hypothesis, we studied the change in compliance as an individual monomer IVVI mutant of the R3 rod domain of talin - a focal adhesion sensor ${ }^{3}$ featuring alpha-helix topology (Fig. 5A) and an associated increment in contour length of $\Delta L \approx 37 \mathrm{~nm}^{26,27}$ (which is significantly larger than that of PL, $\Delta L=18.6 \mathrm{~nm}^{28}$ ) hops in equilibrium between the folded and unfolded states at a constant force of $8.35 \mathrm{pN}^{29}$ (Fig. $5 B \& C)$. Analysis of the hopping trajectories following the same procedure used in Fig. $2 B, C$ significantly larger average increase in protein compliance $\Delta c=1.28 \pm 0.08 \mathrm{~nm} / \mathrm{pN}$ upon unfolding.

\section{The sequential refolding of individual nesprin domains progressively reduce protein fluctuations}

A particular characteristics of the R3 domain of talin is its fast unfolding/dynamics ${ }^{29}$, which implies that at forces only slightly higher (lower) than the hopping $\left(F_{0.5}\right)$ force, the lifetime of the folded (unfolded) state becomes too short-lived to make a thorough measurement of compliance through the length variance (Fig. S5). To circumvent this limitation, we moved to conducting folding measurements on the spectrin73 repeat (SR73) of nesprin, a nuclear envelope mechanosensor protein that has been suggested to play a key role in propagating extracellular mechanical forces to the cell nucleus ${ }^{30}$ likely through mechanical unfolding ${ }^{31}$ and which exhibits a comparable contour length increment $(\Delta L=34.2 \mathrm{~nm}$, Fig. S6C). Stretching an individual [Ig27-SR73] 4 polyprotein (Fig. 6A) in a force-ramp (initial pulse) elicits the stepwise unfolding of the 4 individual SR73 nesprin domains, concomitant to an average increase in the protein length of $\Delta L=28.6 \mathrm{~nm}$ (Fig. 5B, Fig S6A). After complete unfolding of the nesprin SR73 domains with force ramped up to $38 \mathrm{pN}$, the force was then quenched down to a lower value of $7.4 \mathrm{pN}$ to trigger protein folding. We chose this force value because, according to the force-dependent folding probability that we measured for the nesprin SR73 domains (Fig. S6), nesprin folds slow enough to directly capture the independent folding transitions corresponding to the sequential refolding of each individual SR73 monomer. Figure 6B shows how, akin to $\mathrm{PL}^{15}$ and to several domains of titin ${ }^{32}$, the previously mechanically uncharacterised nesprin mechanosensor effectively refolds against a constant force $(7.4 \mathrm{pN})$, marked by the sequential stepwise shrinking $(\Delta\langle x\rangle=16.8 \pm 0.2 \mathrm{~nm})$ of the protein construct. To elucidate whether the sequential stepwise recoil of the protein (Fig. 6C) is mirrored by a step decrease in the end-to-end fluctuation of the polyprotein as each individual monomer refolds, we conducted variance analysis of the refolding trajectory over time and found that, on average ( $n=10$ individual trajectories, Fig. $\mathrm{S} 6 \mathrm{D}, \mathrm{E})$, the folding of each individual domain results in a concomitant change of the construct's compliance by $\Delta c=1.99 \pm 0.12 \mathrm{~nm} / \mathrm{pN}$ (Fig. 6D and Fig. S6D,E). Normalization of the measured change in compliance with the normalised change in extension for proteins with markedly different contour lengths and over a wide range of forces collapses data onto a FJC master curve, further demonstrating that the change in protein compliance is force- and length-dependent (through the FJC relationship) but protein(sequence)- independent (Fig. 5E).

\section{Discussion}

Analysis of molecular fluctuations has enabled capturing the conformational dynamics of proteins using a number of complementary experimental techniques, such as nuclear magnetic resonance (NMR) and small-angle X-ray scattering (SAXS) ${ }^{33,34}$. At the single molecule level, sm-FRET has provided invaluable insight into the broad conformational heterogeneity and dynamics of the unfolded state of proteins, as well as of intrinsically disordered motifs ${ }^{35}$. More recently, interferometric scattering microscopy (iSCAT) has shown its ability to capture conformational transitions, provided they are associated to a change in the protein's center of mass ${ }^{36}$. However, none of these experimental approaches can capture the effect of mechanical load on the conformational dynamics of individual proteins. 
When denatured by force, proteins undergo a conformational change that brings the mechanically stiff, natively folded form into a stretched, compliant and unfolded conformation that cannot be populated in classical biochemical experiments using temperature or chemical denaturants ${ }^{7,37}$. Single molecule nanomechanical techniques, mostly using AFM in the high forceregime $^{7}$ and optical tweezers to probe proteins with lower mechanical stabilities ${ }^{38-40}$, have enabled sampling the regions of the protein free energy landscape that cannot be accessed through any other experimental means, and have rationalized the effect of force on modulating the conformational dynamics of an individual protein during its folding journey.

In this vein, earlier experiments using force-clamp AFM showed that, during collapse from highly-extended states, proteins exhibit increased end-to-end length fluctuations, reminiscent of the temporal occupancy of locally broad energy minima, before reaching their natively folded length $13,24,41,42$. Conformational heterogeneity was also captured at the molten globule ${ }^{43}$ and native states ${ }^{44}$ levels, however these experiments required using the mechanical stability of each conformation as its indirect structural fingerprint. Nonetheless, the high applied forces, the intrinsic stiffness (and mass) of the AFM probe and its associated force uncertainty (and decreased diffusion coefficient ${ }^{20}$ ), in addition to the active PID feedback, have precluded direct capture of the intrinsic fluctuations and underlying stiffness defining each force-dependent protein conformation. Our experiments using magnetic tweezers (where the stiffness of the protein is not masked by the pulling probe) demonstrate that the end-to-end fluctuations of a single protein under force can be used as a direct reporter of its precise conformation and folding status, hence introducing an additional experimentally measurable dimension to the sudden changes in protein length accompanying protein unfolding/refolding events. Noteworthy, these measurements are independent of the mechanical stability, the fold, and the sequence of the studied protein. More importantly, this approach may be of particular relevance to identify time-dependent events occurring without obvious changes in the protein's end-to-end length, such as ligand/drug binding 45 and posttranslational modifications that place dihedral angle constrictions to the force-induced protein conformation ${ }^{46,47}$. This is particularly timely given the fast accumulating evidences that collectively point towards the biological importance underpinning the force-induced exposure of previouslycryptic amino acids ${ }^{5}$, which has been shown to enable post-translational modifications ${ }^{48-50}$ and enzymatic activity ${ }^{47}$ as well as chaperone ${ }^{46}$ and ligand ${ }^{16,51}$ binding. For example, binding of vinculin to unfolded talin ${ }^{52}$ and $\alpha$-catenin ${ }^{16}$ is a central step in cellular mechanotransduction. Importantly, all these functionalities can be finely regulated by the pulling force through precise modulation of protein stiffness and conformation, as revealed, with atomic detail, by steered molecular dynamics (SMD) simulations ${ }^{53}$. It will be enticing to compare, for distinct proteins, how our experimental results agree with the equilibrium fluctuations measured for equivalent force-induced protein conformations in SMD simulations ${ }^{53}$. More broadly, our experiments focusing on the natural fluctuations defining the continuum force-induced thermodynamic equilibrium states complement recent elegant single molecule experiments concentrating on the non-equilibrium transition paths ${ }^{11,54,55}$ between those defined protein states.

\section{Author contributions}

A.S. and S.G.M. designed research. A.E.M.B., M.M. and A.S. conducted experiments, M.L-C. built and A.E.M.B. calibrated the spectrometer, A.S. devised and performed data analysis, S.B. conducted molecular biology and S.G-M. wrote the paper. All authors participated in the discussion and editing of the manuscript.

\section{Acknowledgements}


We thank Julio Fernández for his help in building the magnetic tweezers set-up and for sharing the plasmid for the talin polyprotein. We thank Ainhoa Lezamiz, Palma Rico-Lastres and Aisling Williams for the engineering of the nesprin polyprotein. A.E.M.B. is recipient of a Sir Henry Wellcome fellowship (210887/Z/18/Z). This work was supported by the European Commission (Mechanocontrol, grant agreement 731957), EPSRC Fellowship K00641X/1, Leverhulme Trust Research Leadership Award RL-2016-015, Wellcome Trust Investigator Award 212218/Z/18/Z and Royal Society Wolfson Fellowship RSWF/R3/183006 to S.G.-M. 


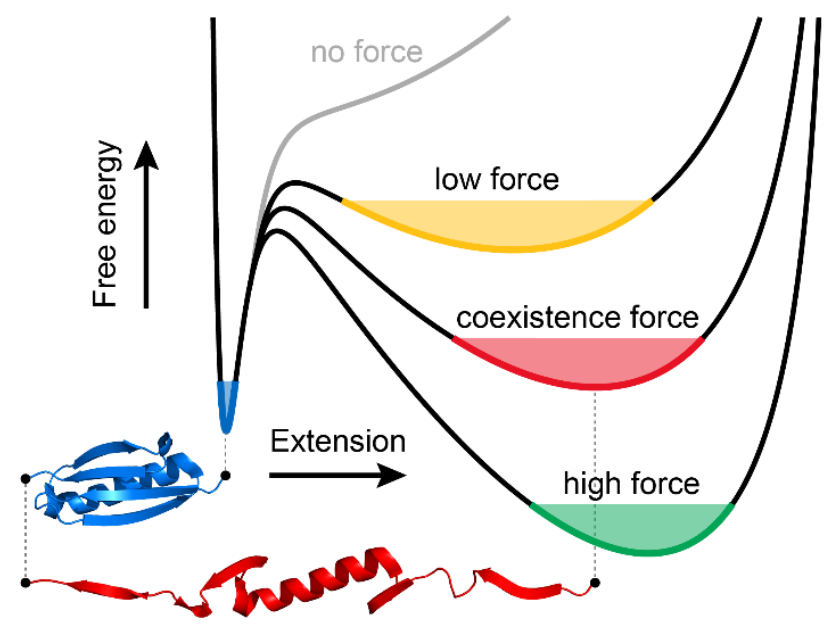

FIG. 1. The effect of force on the free energy landscape of protein L. In the absence of force (grey line), there is one minimum, corresponding to the folded state (blue). When a low force is applied, a broad local minimum corresponding to an unfolded state emerges (yellow). At a certain, coexistence force (red), the protein will equally likely be found in folded and unfolded states. As force increases, the unfolded minimum becomes narrower, eventually becoming the global minimum (green). 
A

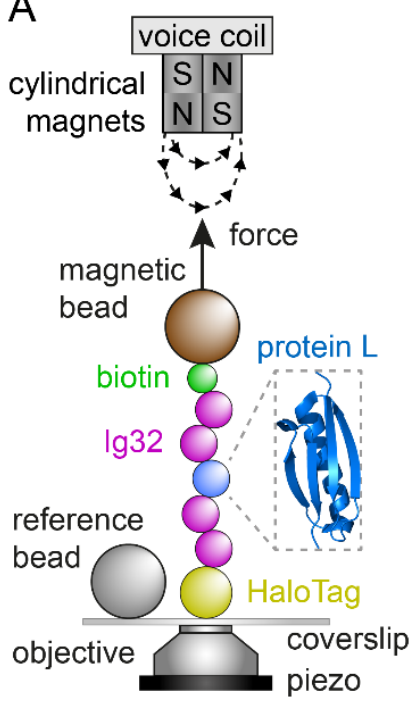

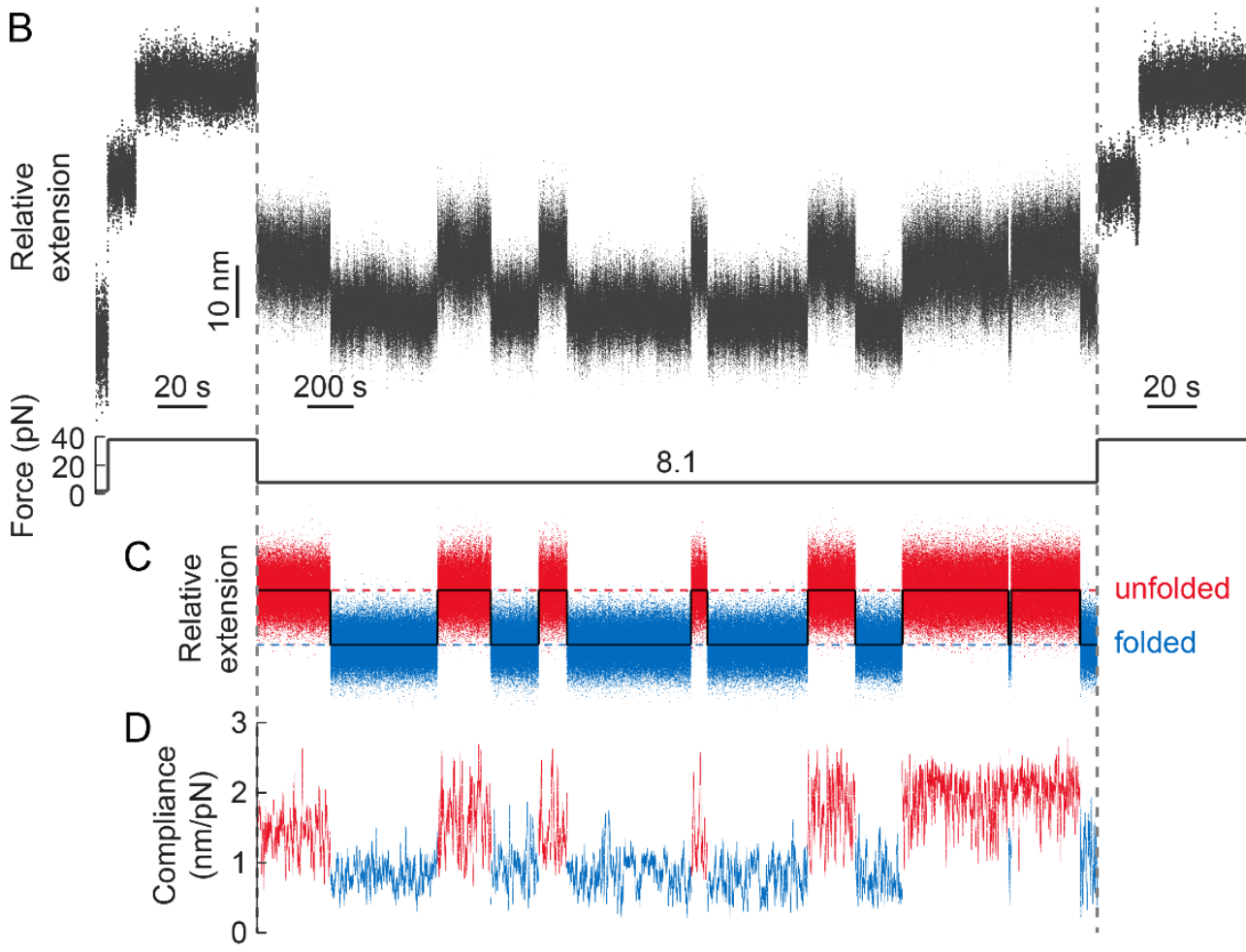

FIG. 2. A step-wise change in protein compliance fingerprints mechanical unfolding/refolding of a single PL monomer under force. (A) Schematic illustration of PL (PDB: 1HZ6) construct being stretched in a magnetic tweezers set-up. (B) Raw extension-time measurement of a protein $L$ monomer hopping in equilibrium between the folded and unfolded states. The $(\operatorname{Ig} 32)_{2}-\mathrm{PL}-(\lg 32)_{2}$ construct is initially pulled at $38 \mathrm{pN}$, which extends and unfolds the PL monomer. After 60s, the force is quenched down to $8.1 \mathrm{pN}$ for 60 minutes. During this time the PL monomer stochastically hops between the folded and unfolded states in steps of $\Delta\langle x\rangle=10.3 \pm 0.3 \mathrm{~nm}$. A final pulse at a high force $(38 \mathrm{pN})$ value triggers the (re)unfolding of the PL monomer. (C) Result of flattening the protein extension data during the $8.1 \mathrm{pN}$ pulse to remove slow drift effects, clearly highlighting 13 individual (un)folding transitions. (D) Compliance of (C) (calculated over a 3-second moving window), showing step-wise changes $(\Delta c=0.86 \pm 0.09 \mathrm{~nm} / \mathrm{pN})$ concomitant to the $\mathrm{PL}$ individual unfolding and refolding events. 


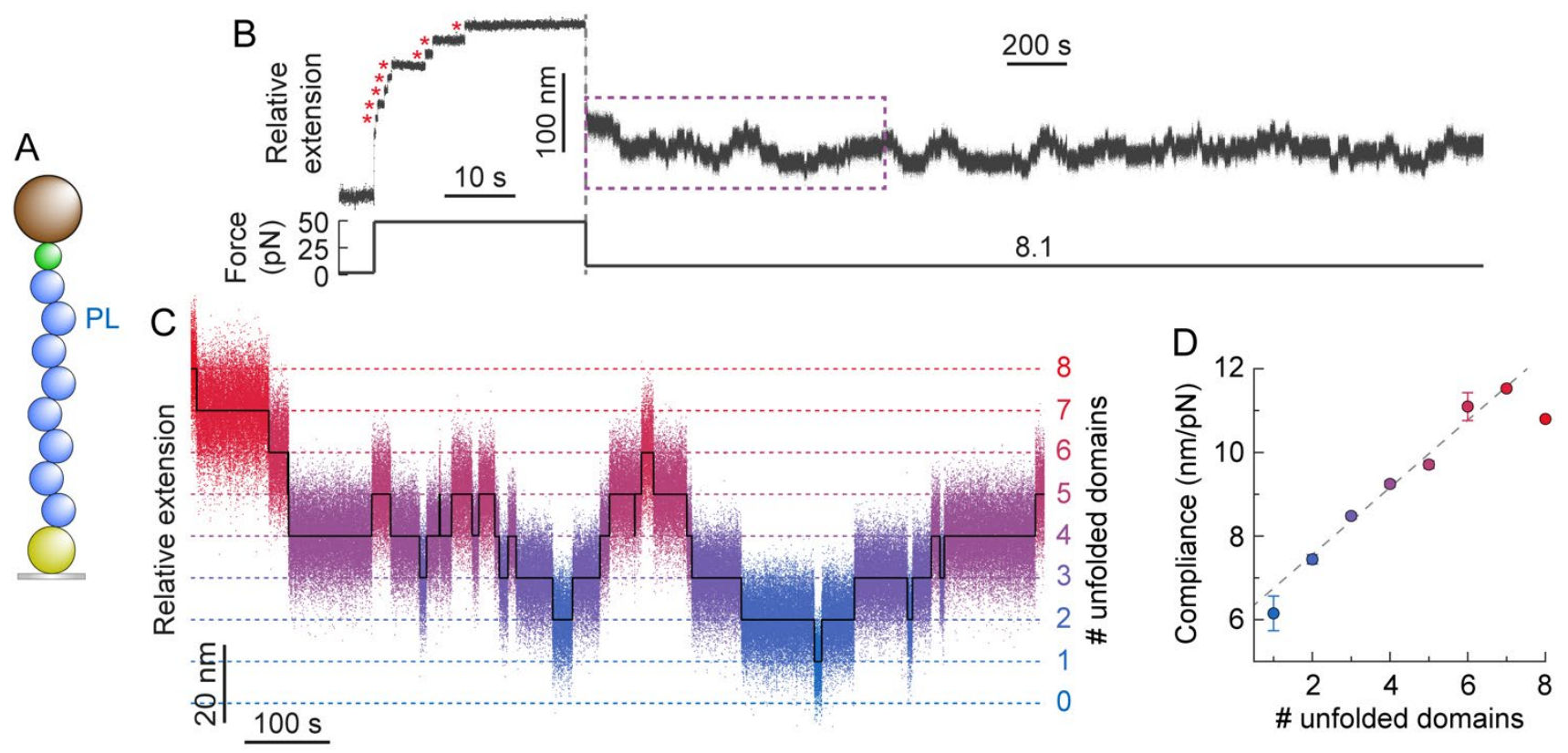

FIG. 3. The change in protein compliance in a $\mathrm{PL}_{8}$ polyprotein is additive and recapitulates that of an individual domain. (A) Illustration of the $\mathrm{PL}_{8}$ construct being pulled in the MT set-up. (B) Typical folding trajectory of $\mathrm{PL}_{8}$ under force. Following a force-quench protocol, a high force pulse (49 $\left.\mathrm{pN}\right)$ is first applied, to trigger the unfolding of each individual domain within the polyprotein chain (red asterisks), hallmarked by changes in extension of $17.2 \mathrm{~nm}$. The force was subsequently dropped down to $8.1 \mathrm{pN}$ (resulting in an initial decrease in extension) and held for 50 minutes. During this period of time, the $\mathrm{PL}_{8}$ polyprotein dynamically hops between well-defined states separated by $\Delta\langle x\rangle=10.7 \pm$ $0.1 \mathrm{~nm}$. (C) Segment flattening and colour-based assignment of the number of unfolded domains allows for clearer visualisation of the step-wise hopping dynamics. (D) The average compliance $(n=$ 131 (un)folding events) can be calculated for a given number of unfolded domains. Linear fit (weighted by the total observation time at each level) to the data yields a gradient of $0.81 \pm 0.15\left(R^{2}\right.$ $=0.983$ ), corresponding to the change in compliance of an individual domain. 


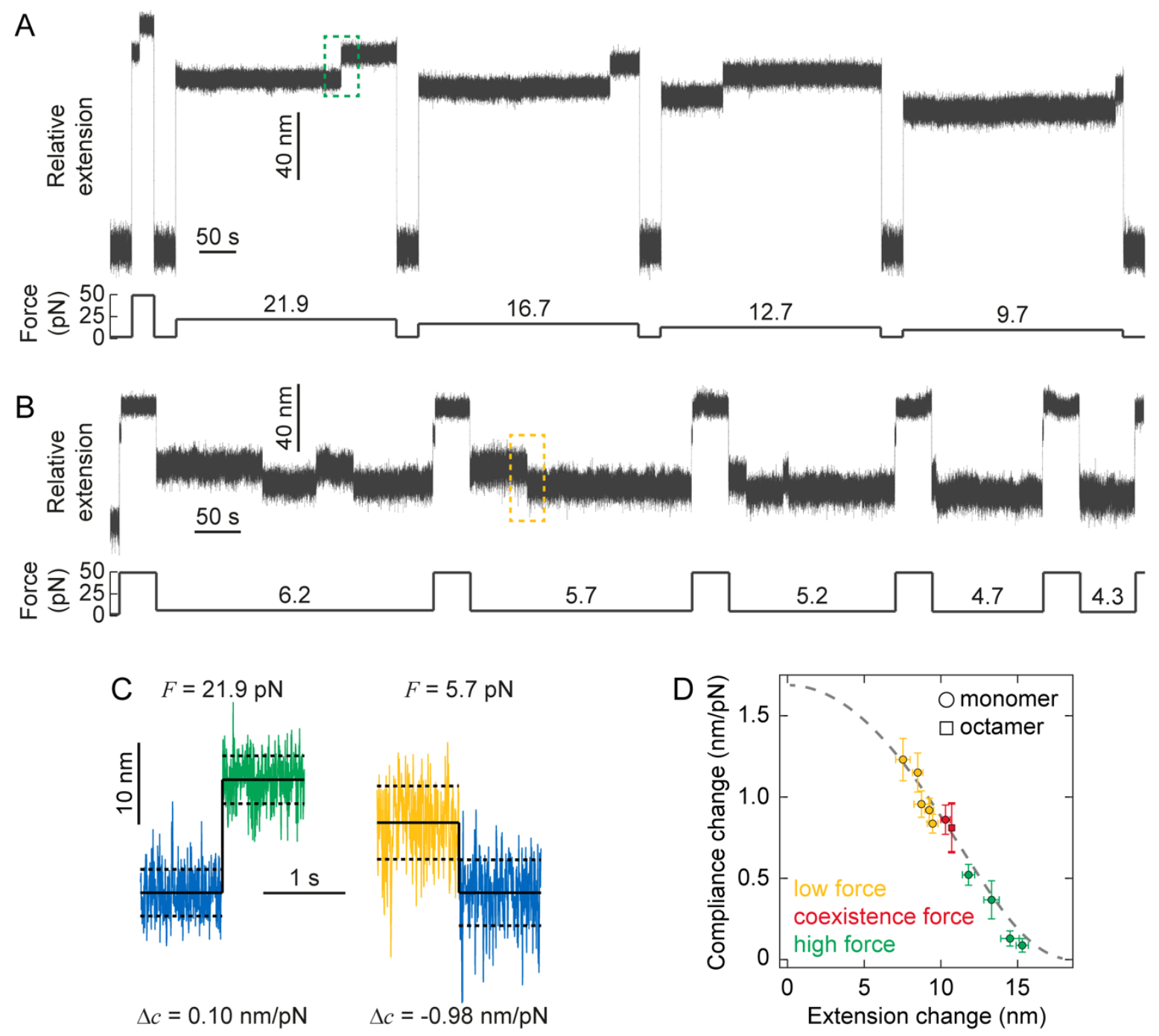

FIG. 4. Correlating force-dependent extension and compliance changes upon mechanical (un)folding through the FJC model of polymer elasticity. (A) Cyclic unfolding and refolding trajectories allow probing the change in protein stiffness upon PL unfolding at varying high forces. Using a force quench protocol, the protein is initially held at a seemingly low force (1.8 pN) for 30 seconds to ensure correct refolding. The force is then increased to a higher constant force value in each cycle (namely 21.9, 16.7, 12.7, and $9.7 \mathrm{pN}$ ) for 5 minutes, which triggers in each case the unfolding of the PL monomer (marked by a step-wise increase in length). (B) Similar cyclic forcequench protocol to capture the change in protein compliance upon mechanical refolding at low forces. Before each cycle, the force is first kept low $(1.8 \mathrm{pN})$ for 30 seconds to reach the protein's native state. Subsequently, in each cycle the protein is unfolded at high force $(49.2 \mathrm{pN})$ for 40 seconds, before the force is successively quenched at low forces, namely $6.2 \mathrm{pN}(5 \mathrm{~min}), 5.7 \mathrm{pN}(4$ $\mathrm{min}), 5.2 \mathrm{pN}(3 \mathrm{~min}), 4.7 \mathrm{pN}(2 \mathrm{~min})$ and $4.3 \mathrm{pN}(1 \mathrm{~min})$, respectively. When held at such low force values, the protein stochastically refolds back to the native state, as evidenced by the step-wise reduction in length. While the folded state dominates at these forces, some hopping back to an unfolded state is observed. (C) Representative unfolding (left) and folding (right) events occurring at high $(21.9 \mathrm{pN})$ and low $(5.7 \mathrm{pN})$ forces respectively (corresponding to the green dashed box in $(\mathrm{A})$ and the yellow dashed box in (B), respectively). High force unfolding is hallmarked by a large increase in average extension but a small increase in compliance, conversely low force folding is hallmarked by a small decrease in average extension but a large decrease in compliance. (D) Plot of (absolute) 
compliance change against (absolute) extension change combining low (yellow), hopping (red), and high (green) force experiments, clearly showing that, upon PL un/folding, an inverse correlation between its changes in extension and compliance is measured. Data points for monomers (circles) correspond to the mean \pm s.e. of 9-17 events occurring at each force, data for the $\mathrm{PL}_{8}$ octamer (square) is taken from Fig. 3D. Combined, this data show very good agreement with the FJC model (grey dashed line) with Kuhn length $b=1.1 \mathrm{~nm}, k_{B} T=4.04 \mathrm{pN} \mathrm{nm}$, and $\Delta L=18.6 \mathrm{~nm}$. 

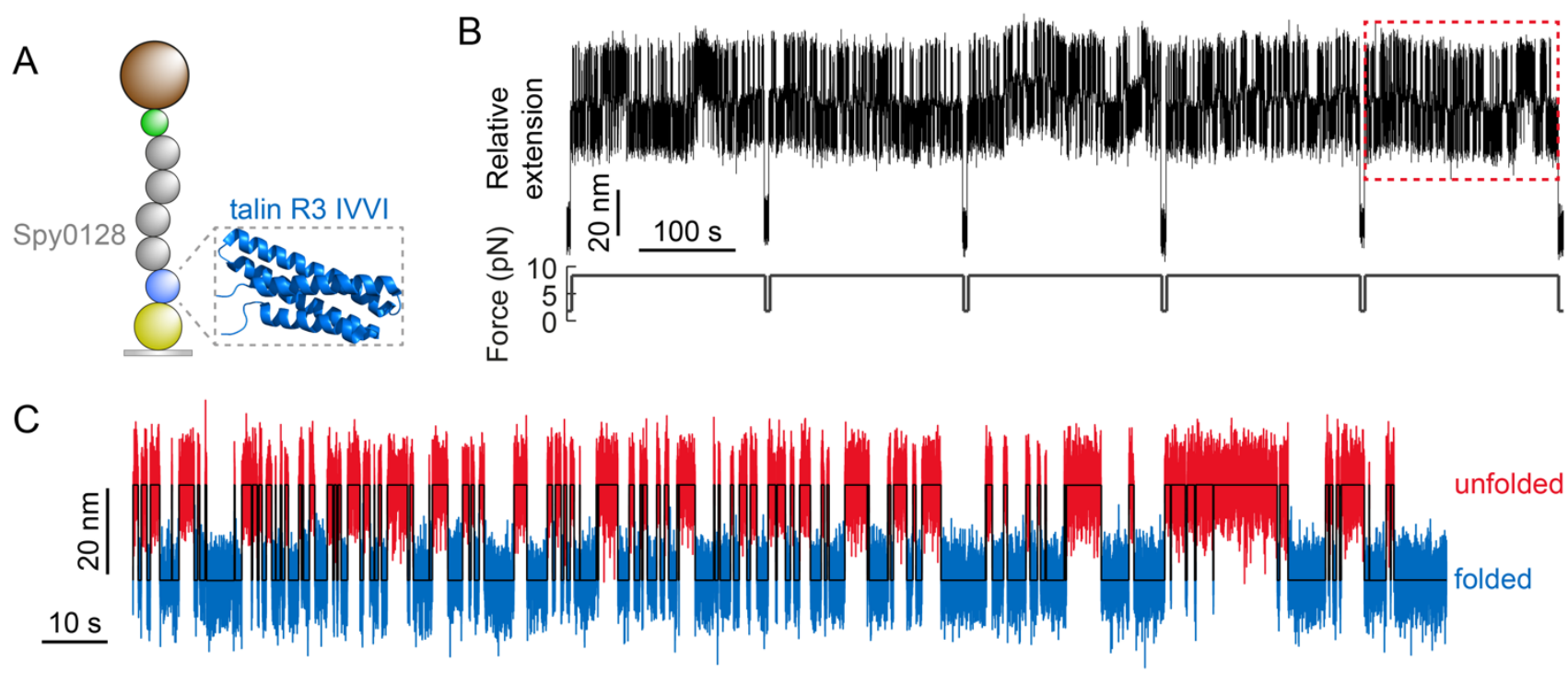

FIG. 5. The equilibrium hopping of the mechanosensitive R3 IVVI domain of talin is directly hallmarked by a change in protein compliance. (A) Illustration of the [(Spy0128) $)_{2}$-TalinR3 $\left.3_{\text {IVvI }}\right]$ construct being pulled in the MT set-up (PDB: 2L7A). (B) Raw, unfiltered extension-time data corresponding to 5 cycles consisting of stretching the individual talin R3 IVVI monomer at a constant force of $8.35 \mathrm{pN}$ for 200 seconds. During this time, the protein monomer undergoes multiple unfolding and refolding transitions. Between two contiguous cycles, the force is further quenched down to $1.8 \mathrm{pN}$ to ensure that the protein is properly refolded. (C) Zoom in on the hopping section highlighted in red in (B), showing 178 consecutive unfolding/refolding transitions concomitant to an average change in extension of $\Delta\langle x\rangle=22.2 \pm 0.2 \mathrm{~nm}$, and an average change in compliance between the folded and unfolded states of $\Delta c=1.24 \pm 0.14 \mathrm{~nm} / \mathrm{pN}$. These values are representative of those obtained by averaging over the 5 cycles of $200 \mathrm{~s}$, where we find $\Delta\langle x\rangle=22.2 \pm 0.1 \mathrm{~nm}$ and $\Delta c=1.28$ $\pm 0.08 \mathrm{~nm} / \mathrm{pN}$. 
A

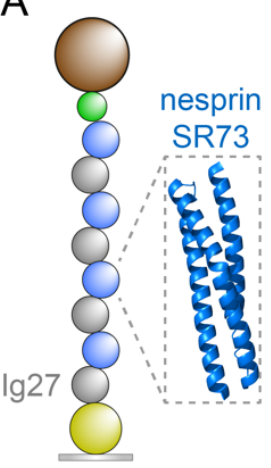

C

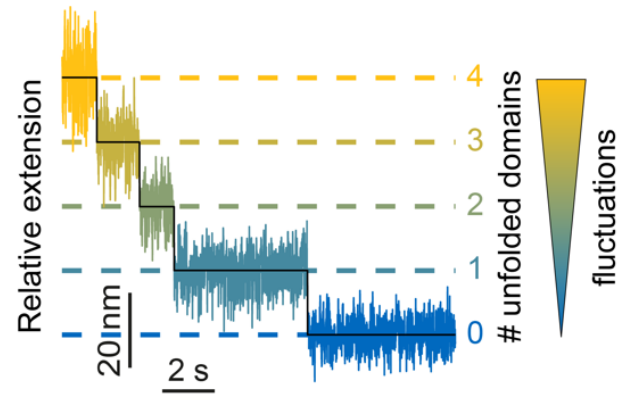

B
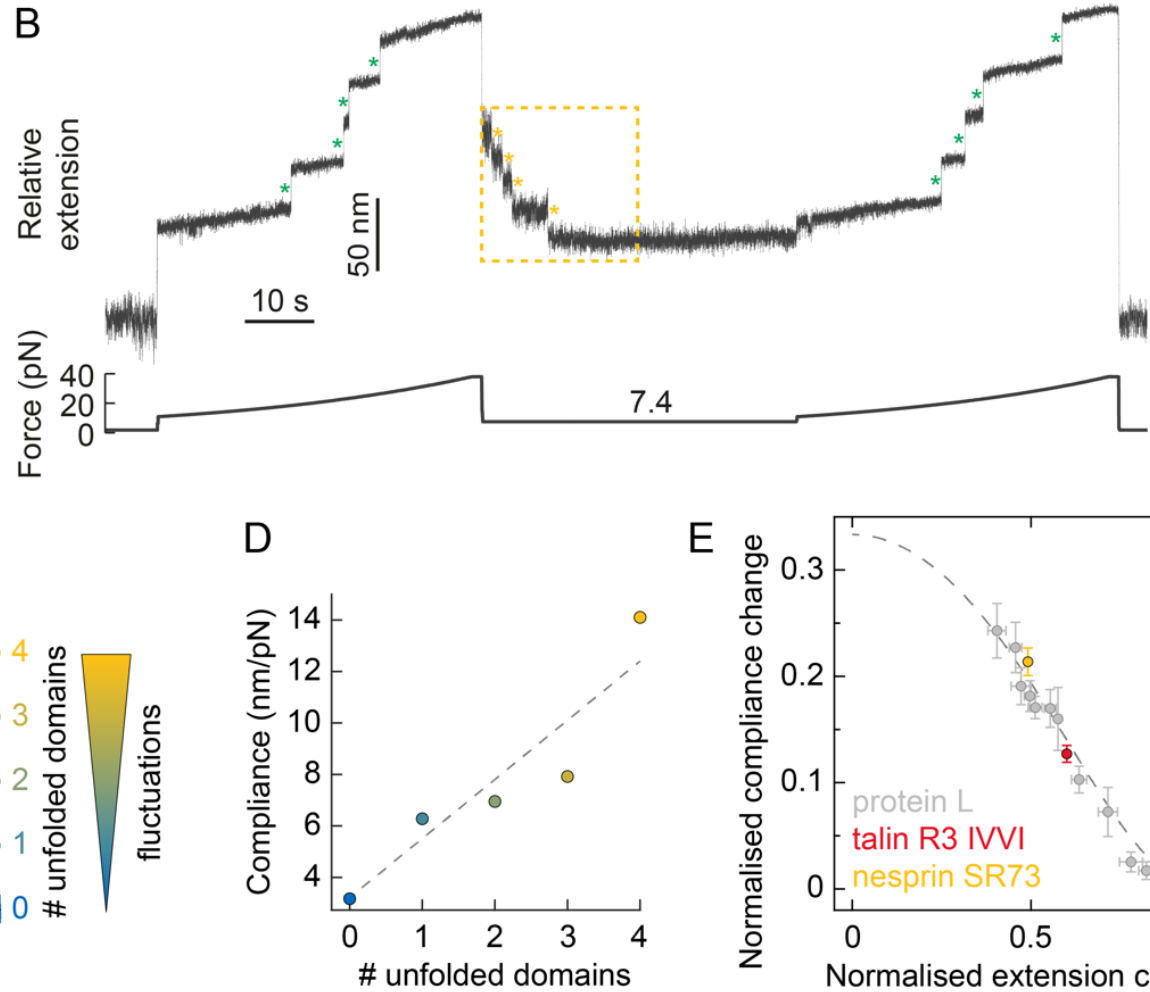

E

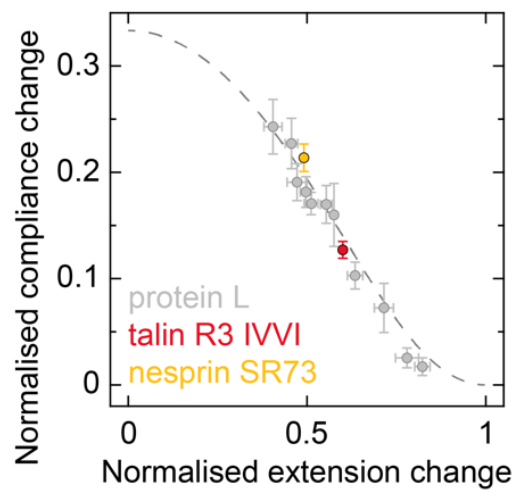

FIG. 6. Direct observation of the change in end-to-end fluctuations upon individual nesprin refolding events. (A) Illustration of the [Ig27-SR73 $]_{4}$ construct being pulled in the MT set-up (lacking crystal structure, here we display the similar SR16 (PDB: 1U4Q). (B) Typical individual (unfiltered) folding trajectory under a force quench. The force is first increased from $10.6 \mathrm{pN}$ to $37.5 \mathrm{pN}$ over the course of $30 \mathrm{~s}$ (corresponding to the linear movement of magnet position by $1.4 \mathrm{~mm}$ over this time), resulting in the sequential step-wise unfolding of the four SR73 domains of nesprin (blue asterisks). Note that, within this force range, the mechanically rigid Ig27 domains in the construct do not unfold. The force is then quenched down to $7.4 \mathrm{pN}$ for 30 seconds, triggering the collapse and refolding of nesprin. At such low $\left(<F_{0.5}\right)$ force value, refolding was slowed down, featuring a refolding staircaselike pattern that mirrored the unfolding one. The force is subsequently ramped up again (test pulse) back to $37.5 \mathrm{pN}$, to probe that all the individual SR73 domains (blue asterisks) had effectively refolded during the force-quench. (C) Zoom (yellow square) on the initial folding section of (B), where the four SR73 domains of nesprin refold sequentially, marked by a step-wise reduction of the protein extension by $\Delta\langle x\rangle=17.1 \pm 0.5 \mathrm{~nm}$. The refolding of each individual domain occurs concomitant to a reduction in the end-to-end fluctuations of the protein construct. (D) Plotting the compliance against the number of unfolded domains measured in (C) allows calculation of the change in compliance per domain, $\Delta c /$ domain $=2.30 \pm 0.59 \mathrm{~nm} / \mathrm{pN}$. Averaging over 10 such folding trajectories with four SR73 nesprin unfolded domains gives $\Delta\langle x\rangle=16.8 \pm 0.2 \mathrm{~nm}$ and $\Delta c /$ domain $=1.99 \pm 0.12$ $\mathrm{nm} / \mathrm{pN}$. (E) Plotting normalised compliance change $(\Delta c \cdot k T / b \Delta L)$ against normalised extension change $(\Delta x / \Delta L)$ crucially reveals that the observed behaviour is protein independent, showing quantitative agreement with a master curve of the FJC model. 


\section{Materials and methods}

\section{Polyprotein engineering}

The Ig32 protein monomer was created by Thermofisher Scientific GenArt ${ }^{\mathrm{TM}}$ with incorporated BamHI, BgllI and KpnI restriction sites and subcloned into pQE80L vector (Qiagen). (Ig32) 2 -PL-(Ig32) 2 and (Ig27-SR73) ${ }_{4}$ polymers were engineered by restriction digest using the compatible cohesive end restriction enzymes BamHI and BgIII between BamHI and Kpnl sites. pFN18a talin R3 IVVI-Spy0128 and PQE80L $\mathrm{PL}_{8}$ were kindly gifted from Julio Fernandez. PQE80L (Ig27-SR73) 4 , PQE80L PL8 and pQE80L Ig $32_{2}-\mathrm{PL}-\lg 32_{2}$ were subcloned into a modified pFN18a vector engineered with the AviTag ${ }^{\mathrm{TM}}$ (avidity) (sequence GLNDIFEAQKIEWHE). Constructs were cloned between the HaloTag at the Nterminus and the $6 \mathrm{x}$ histidine tag adjacent to the AviTag ${ }^{\mathrm{TM}}$ at the C-terminus. Recombinant plasmids were transformed in XL1Blue (Agilent Technologies) or Top10 (Thermofisher scientific) competent cells. Selected colonies were grown in Luria broth supplemented with $100 \mathrm{mg} / \mathrm{ml}$ ampicillin at $37^{\circ} \mathrm{C}$. Cells were lysed and plasmid DNA was purified before transfection using a Qiagen kit according to the manufacturer's instructions.

Constructs were expressed in E. coli BLR(D3) cells (Novagen). Cells were grown in Luria broth supplemented with $100 \mathrm{mg} / \mathrm{ml}$ ampicillin at $37^{\circ} \mathrm{C}$. After reaching an $\mathrm{OD}_{600}$ of about 0.6 , cultures were induced with $1 \mathrm{mM}$ isopropyl- $\beta$-D-thiogalactopyranoside and grown at $25^{\circ} \mathrm{C}$ for $16 \mathrm{~h}$. Cells were resuspended in buffer $50 \mathrm{mM}$ sodium phosphate $(\mathrm{NaPi}) \mathrm{pH} 7.0,300 \mathrm{mM} \mathrm{NaCl}, 10 \%$ glycerol and $1 \mathrm{mM}$ DTT supplemented with $100 \mathrm{mg} / \mathrm{ml}$ lysozyme, $5 \mu \mathrm{g} / \mathrm{ml}$ DNase, $5 \mu \mathrm{g} / \mathrm{ml} \mathrm{RNase}$ and $10 \mathrm{mM} \mathrm{MgCl} 2$ and incubated on ice for 30 minutes. Cells were disrupted by a French press and lysate was centrifuged at 20,000 $\mathrm{g}$ for 40 minutes before purification by Talon affinity resin (Clontech) using wash buffer (50 $\mathrm{mM} \mathrm{NaPi} \mathrm{pH} \mathrm{7.0,300} \mathrm{mM} \mathrm{NaCl,} \mathrm{10 \%} \mathrm{glycerol,} 1 \mathrm{mM}$ DTT, $20 \mathrm{mM}$ imidazole). Protein was eluted with $250 \mathrm{mM}$ imidazole. This was followed by gel filtration using a Superdex 200 10/300 GL column (GE Biosciences). Proteins were stored in $10 \mathrm{mM}$ HEPES pH 7.2, $150 \mathrm{mM} \mathrm{NaCl}, 10 \%$ glycerol, $1 \mathrm{mM}$ EDTA at $-20^{\circ} \mathrm{C}$.

Gel filtration fractions were pooled and concentrated using Amicon ${ }^{\circledR}$ filters with selected MWCO. Constructs were biotinylated using BirA Biotin Ligase (Avidity) following the suggested protocol ( $40 \mu \mathrm{M}$ substrate, $1 \mathrm{x}$ buffer $\mathrm{A}, 1 \mathrm{x}$ buffer $\mathrm{B}, 2.5 \mu \mathrm{g}$ enzyme BirA, at a final volume of $200 \mu \mathrm{l}$ ). The reaction was left for 3 hours at $30^{\circ} \mathrm{C}$ ). Biotinylation was confirmed using Streptavidin HRP conjugate (Millipore) with biotinylated/unbiotinylated MBP-AviTag ${ }^{\mathrm{TM}}$ fusion protein (Avidity) as controls.

\section{Single-molecule magnetic tweezers experiments}

Sample preparation and the single-molecule magnetic tweezers setup are based on those reported elsewhere ${ }^{15}$, with slight variations. Briefly, the single-molecule experiments were performed on a custom-made setup built on top of an inverted microscope (Nikon Eclipse TE2000-S) and using a 100x oil-immersion objective mounted on a high-precision positioner actuator (P-725 PIFOC objective scanner, Physik Instrumente). The sample was illuminated with white light (M590L3-C5, Thorlabs) and images were acquired using a CCD camera with $2 \mu$ s exposure time and a frame rate of $\sim 280 \mathrm{fps}$ (Pike F-421, Allied Vision Technologies). The position of the permanent magnets (D33-N52, K\&J Magnetics) was precisely controlled by a linear voice-coil (LFA-2010, Equipment Solutions) with 150 $\mathrm{nm}$ position resolution.

Single molecule experiments were performed in fluid chambers composed of two cover slides ( $40 \times 24 \mathrm{~mm}$ and $22 \times 22 \mathrm{~mm}$, bottom and top respectively, thickness \#1, Agar Scientific) with parafilm sandwiched in between. To construct these chambers, first, all cover slides were cleaned by sonication; with $1 \%$ Hellmanex solution $\left(60{ }^{\circ} \mathrm{C}\right.$ for 30 minutes), then with acetone (R.T. for 30 
minutes), and finally with ethanol (R.T. for 30 minutes), before drying at $100{ }^{\circ} \mathrm{C}$ for 30 minutes. In order to eliminate reflections from the magnetic faces located above the fluid chamber, the top surfaces of the top cover slides were coated with matt black spray paint (Rust-Oleum). The bottom cover slides were first activated by plasma cleaning (20 minutes) before silanization (immersion in $0.1 \% \mathrm{v} / \mathrm{v} 3$-(aminopropyl)trimethoxysilane in methanol for 30 minutes). The silanized cover slides were then cured at $100^{\circ} \mathrm{C}$ for 30 minutes. Fluid chambers were assembled by placing a parafilm cutouts ( $40 \times 24 \mathrm{~mm}$ with the central $\sim 30 \times 16 \mathrm{~mm}$ removed) between top and bottom cover slides followed by brief heating on a hot plate at $100^{\circ} \mathrm{C}$ to ensure adhesion. After assembly, chambers were incubated with an aqueous glutaraldehyde solution (glutaraldehyde grade $170 \% ; 0.01 \% \mathrm{v} / \mathrm{v}$ ) for 60 minutes, followed by incubation with $0.02 \% \mathrm{w} / \mathrm{v}$ polystyrene beads/microspheres $(\sim 2.68 \mu \mathrm{m}$ diameter, Spherotech). After 20 minutes, those beads unattached to chamber bottoms were washed out with PBS. Chambers were then incubated with a $10 \mu \mathrm{g} / \mathrm{ml}$ solution of the HaloTag amine (04) ligand (Promega) in PBS, and left overnight in a humidity chamber. Finally, chambers were filled with a BSA-blocking buffer (20 mM Tris- $\mathrm{HCL}$ pH 7.3, $150 \mathrm{mM} \mathrm{NaCl}, 2 \mathrm{mM} \mathrm{MgCl}$ and $1 \% \mathrm{w} / \mathrm{v}$ sulfhydryl blocked BSA, MyBioSource), for a minimum of 12 hours before use. Once fluid chambers were ready, polyprotein constructs were freshly diluted in PBS buffer to a concentration $\sim 0.01-0.02 \mathrm{nM}$ and incubated in a chamber for 30 minutes to achieve HaloTag binding. Unreacted polyproteins were washed out with PBS. Finally, streptavidin-coated paramagnetic beads (Dynabeads M-270, Invitrogen) were added to the chamber to bind with the biotinylated polyprotein terminus for $\sim 10$ min. Unbound beads were then washed out with a $5 \mathrm{mM}$ ascorbic acid solution in PBS (pH 7.3).

\section{Compliance calculation from the freely-jointed chain (FJC) model of polymer elasticity}

The freely jointed chain (FJC) is a powerful way to describe the mechanical behaviour of polymers, when treated as an ideal chain. An FJC consists of $n$ rigid segments of fixed length, $b$ - the Kuhn length - connected via free joints (no steric hinderance/restrictions in either radial or azimuthal angles between adjacent segments). Each of these Kuhn segments represents a section of polymer that can be approximated to be behaving as an independent, discrete unit. The contour length (maximum end-to-end separation) of an FJC is simply given by $L=n b$, and it is a well-known result that the partition function of an FJC under the constraint of a constant tensile force $F$ is

$$
Z=\left[4 \pi(\beta b F)^{-1} \sinh (\beta b F)\right]^{n}
$$

Above we showed in the previous section that $\partial_{F} Z=\beta Z\langle x\rangle$ for any ideal chain, thus

$$
\langle x\rangle=(\beta Z)^{-1} \partial_{F} Z=\beta^{-1} \partial_{F}(\ln Z)
$$

and for the FJC model the force-dependent average extension is

$$
\langle x\rangle=\beta^{-1} n \partial_{F}\left\{\ln \left[4 \pi(\beta b F)^{-1} \sinh (\beta b F)\right]\right\}=L\left[\operatorname{coth}(\beta b F)-(\beta b F)^{-1}\right]
$$

where the function in squared brackets can be recognised as the Langevin function of the dimensionless force $\beta b F$.

Since $c \equiv \partial_{F}\langle x\rangle$, an expression for the force-dependent compliance of an FJC is then

$$
c=\beta b L\left[1-\operatorname{coth}^{2}(\beta b F)-(\beta b F)^{-2}\right]
$$

It is relatively trivial, but time-consuming, to show that this same result can be obtained by using $c=$ $\beta \sigma^{2}=\beta\left\langle x^{2}\right\rangle-\beta\langle x\rangle^{2}$ after explicitly finding $\langle x\rangle=(\beta Z)^{-1} \partial_{F} Z$ and $\left\langle x^{2}\right\rangle=\beta^{-2} Z^{-1} \partial_{F}^{2} Z$ from the FJC partition function. 


\section{References}

1. Rief, M., Gautel, M., Oesterhelt, F., Fernandez, J.M. \& Gaub, H.E. Reversible unfolding of individual titin immunoglobulin domains by AFM. Science 276, 1109-12 (1997).

2. Elosegui-Artola, A. et al. Mechanical regulation of a molecular clutch defines force transmission and transduction in response to matrix rigidity. Nat Cell Biol 18, 540-8 (2016).

3. Yao, M. et al. The mechanical response of talin. Nat Commun 7, 11966 (2016).

4. Infante, E. et al. The mechanical stability of proteins regulates their translocation rate into the cell nucleus. Nature Physics 15, 973-981 (2019).

5. Vogel, V. Mechanotransduction involving multimodular proteins: converting force into biochemical signals. Annu Rev Biophys Biomol Struct 35, 459-88 (2006).

6. Cao, Y. \& Li, H. Engineered elastomeric proteins with dual elasticity can be controlled by a molecular regulator. Nat Nanotechnol 3, 512-6 (2008).

7. Mora, M., Stannard, A. \& Garcia-Manyes, S. The nanomechanics of individual proteins. Chem Soc Rev (2020).

8. Garcia-Manyes, S. \& Beedle, A.E.M. Steering chemical reactions with force. Nature Reviews Chemistry 1(2017).

9. Schonfelder, J., Alonso-Caballero, A., De Sancho, D. \& Perez-Jimenez, R. The life of proteins under mechanical force. Chem Soc Rev 47, 3558-3573 (2018).

10. Puchner, E.M. \& Gaub, H.E. Force and function: probing proteins with AFM-based force spectroscopy. Curr Opin Struct Biol 19, 605-14 (2009).

11. Cossio, P., Hummer, G. \& Szabo, A. Transition paths in single-molecule force spectroscopy. J Chem Phys 148, 123309 (2018).

12. Smith, S.B., Cui, Y. \& Bustamante, C. Overstretching B-DNA: the elastic response of individual double-stranded and single-stranded DNA molecules. Science 271, 795-9 (1996).

13. Berkovich, R., Garcia-Manyes, S., Urbakh, M., Klafter, J. \& Fernandez, J.M. Collapse dynamics of single proteins extended by force. Biophys J 98, 2692-701 (2010).

14. Lof, A. et al. Multiplexed protein force spectroscopy reveals equilibrium protein folding dynamics and the low-force response of von Willebrand factor. Proc Natl Acad Sci U S A 116, 18798-18807 (2019).

15. Popa, I. et al. A HaloTag Anchored Ruler for Week-Long Studies of Protein Dynamics. J Am Chem Soc 138, 10546-53 (2016).

16. Yao, M. et al. Force-dependent conformational switch of alpha-catenin controls vinculin binding. Nat Commun 5, 4525 (2014).

17. Guo, Z. et al. Hidden Intermediate State and Second Pathway Determining Folding and Unfolding Dynamics of GB1 Protein at Low Forces. Phys Rev Lett 125, 198101 (2020).

18. Rico-Pasto, M., Pastor, I. \& Ritort, F. Force feedback effects on single molecule hopping and pulling experiments. J Chem Phys 148, 123327 (2018).

19. Hinczewski, M., Gebhardt, J.C., Rief, M. \& Thirumalai, D. From mechanical folding trajectories to intrinsic energy landscapes of biopolymers. Proc Natl Acad Sci U S A 110, 4500-5 (2013).

20. Berkovich, R. et al. Rate limit of protein elastic response is tether dependent. Proc Natl Acad Sci U S A 109, 14416-21 (2012).

21. De Sancho, D., Schonfelder, J., Best, R.B., Perez-Jimenez, R. \& Munoz, V. Instrumental Effects in the Dynamics of an Ultrafast Folding Protein under Mechanical Force. J Phys Chem B 122, 11147-11154 (2018). 
22. Cossio, P., Hummer, G. \& Szabo, A. On artifacts in single-molecule force spectroscopy. Proc Natl Acad Sci U S A 112, 14248-53 (2015).

23. Walther, K.A., Brujic, J., Li, H. \& Fernandez, J.M. Sub-angstrom conformational changes of a single molecule captured by AFM variance analysis. Biophys J 90, 3806-12 (2006).

24. Garcia-Manyes, S., Brujic, J., Badilla, C.L. \& Fernandez, J.M. Force-clamp spectroscopy of single-protein monomers reveals the individual unfolding and folding pathways of $\mathrm{I} 27$ and ubiquitin. Biophys J 93, 2436-46 (2007).

25. Lei, H. et al. Single-Molecule Force Spectroscopy Trajectories of a Single Protein and Its Polyproteins Are Equivalent: A Direct Experimental Validation Based on A Small Protein NuG2. Angew Chem Int Ed Engl 56, 6117-6121 (2017).

26. Haining, A.W., von Essen, M., Attwood, S.J., Hytonen, V.P. \& Del Rio Hernandez, A. All Subdomains of the Talin Rod Are Mechanically Vulnerable and May Contribute To Cellular Mechanosensing. ACS Nano 10, 6648-58 (2016).

27. Tapia-Rojo, R., Alonso-Caballero, A. \& Fernandez, J.M. Direct observation of a coil-to-helix contraction triggered by vinculin binding to talin. Sci Adv 6, eaaz4707 (2020).

28. Brockwell, D.J. et al. Mechanically unfolding the small, topologically simple protein L. Biophys J 89, 506-19 (2005).

29. Tapia-Rojo, R., Alonso-Caballero, A. \& Fernandez, J.M. Talin folding as the tuning fork of cellular mechanotransduction. Proc Natl Acad Sci U S A 117, 21346-21353 (2020).

30. Rajgor, D. \& Shanahan, C.M. Nesprins: from the nuclear envelope and beyond. Expert Rev Mol Med 15, e5 (2013).

31. Arsenovic, P.T. et al. Nesprin-2G, a Component of the Nuclear LINC Complex, Is Subject to Myosin-Dependent Tension. Biophys J 110, 34-43 (2016).

32. Rivas-Pardo, J.A. et al. Work Done by Titin Protein Folding Assists Muscle Contraction. Cell Rep 14, 1339-1347 (2016).

33. Ishima, R. \& Torchia, D.A. Protein dynamics from NMR. Nat Struct Biol 7, 740-3 (2000).

34. Fuertes, G. et al. Decoupling of size and shape fluctuations in heteropolymeric sequences reconciles discrepancies in SAXS vs. FRET measurements. Proc Natl Acad Sci U S A 114, E6342-E6351 (2017).

35. Schuler, B., Soranno, A., Hofmann, H. \& Nettels, D. Single-Molecule FRET Spectroscopy and the Polymer Physics of Unfolded and Intrinsically Disordered Proteins. Annu Rev Biophys 45, 207-31 (2016).

36. Andrecka, J. et al. Structural dynamics of myosin 5 during processive motion revealed by interferometric scattering microscopy. Elife 4(2015).

37. Walther, K.A. et al. Signatures of hydrophobic collapse in extended proteins captured with force spectroscopy. Proc Natl Acad Sci U S A 104, 7916-21 (2007).

38. Cecconi, C., Shank, E.A., Bustamante, C. \& Marqusee, S. Direct observation of the threestate folding of a single protein molecule. Science 309, 2057-60 (2005).

39. Shank, E.A., Cecconi, C., Dill, J.W., Marqusee, S. \& Bustamante, C. The folding cooperativity of a protein is controlled by its chain topology. Nature 465, 637-40 (2010).

40. Stigler, J., Ziegler, F., Gieseke, A., Gebhardt, J.C. \& Rief, M. The complex folding network of single calmodulin molecules. Science 334, 512-6 (2011).

41. Fernandez, J.M. \& Li, H. Force-clamp spectroscopy monitors the folding trajectory of a single protein. Science 303, 1674-8 (2004).

42. Lannon, H., Haghpanah, J.S., Montclare, J.K., Vanden-Eijnden, E. \& Brujic, J. Force-clamp experiments reveal the free-energy profile and diffusion coefficient of the collapse of protein molecules. Phys Rev Lett 110, 128301 (2013). 
43. Garcia-Manyes, S., Dougan, L., Badilla, C.L., Brujic, J. \& Fernandez, J.M. Direct observation of an ensemble of stable collapsed states in the mechanical folding of ubiquitin. Proc Natl Acad Sci U S A 106, 10534-9 (2009).

44. Brujić, J., Hermans Z, R.I., Walther, K.A. \& Fernandez, J.M. Single-molecule force spectroscopy reveals signatures of glassy dynamics in the energy landscape of ubiquitin. Nature Physics 2, 282-286 (2006).

45. Gupta, A.N. et al. Pharmacological chaperone reshapes the energy landscape for folding and aggregation of the prion protein. Nat Commun 7, 12058 (2016).

46. Perales-Calvo, J., Giganti, D., Stirnemann, G. \& Garcia-Manyes, S. The force-dependent mechanism of DnaK-mediated mechanical folding. Sci Adv 4, eaaq0243 (2018).

47. Wiita, A.P. et al. Probing the chemistry of thioredoxin catalysis with force. Nature 450, 124-7 (2007).

48. Beedle, A.E., Lynham, S. \& Garcia-Manyes, S. Protein S-sulfenylation is a fleeting molecular switch that regulates non-enzymatic oxidative folding. Nat Commun 7, 12490 (2016).

49. Valle-Orero, J. et al. Proteins Breaking Bad: A Free Energy Perspective. J Phys Chem Lett 8, 3642-3647 (2017).

50. Loescher, C.M. et al. Regulation of titin-based cardiac stiffness by unfolded domain oxidation (UnDOx). Proc Natl Acad Sci U S A 117, 24545-24556 (2020).

51. Yao, M. et al. Mechanical activation of vinculin binding to talin locks talin in an unfolded conformation. Sci Rep 4, 4610 (2014).

52. del Rio, A. et al. Stretching single talin rod molecules activates vinculin binding. Science 323, 638-41 (2009).

53. Stirnemann, G., Giganti, D., Fernandez, J.M. \& Berne, B.J. Elasticity, structure, and relaxation of extended proteins under force. Proc Natl Acad Sci U S A 110, 3847-52 (2013).

54. Neupane, K. et al. Direct observation of transition paths during the folding of proteins and nucleic acids. Science 352, 239-42 (2016).

55. Neupane, K., Manuel, A.P. \& Woodside, M.T. Protein folding trajectories can be described quantitatively by one-dimensional diffusion over measured energy landscapes. Nature Physics 12, 700-703 (2016). 